\title{
A short derivation of the Möbius function for the Bruhat order
}

\author{
John R. Stembridge
}

Received: 10 March 2006 / Accepted: 26 June 2006 /

Published online: 22 July 2006

(C) Springer Science + Business Media, LLC 2007

\begin{abstract}
We give a short, self-contained derivation of the Möbius function for the Bruhat orderings of Coxeter groups and their parabolic quotients.
\end{abstract}

Keywords Coxeter group $\cdot$ Bruhat order $\cdot$ Möbius function

\section{Introduction}

The Bruhat orderings of Coxeter groups and their parabolic quotients play a significant role in representation theory and related geometry, primarily due to the fact that in special cases, these partial orderings encode the inclusions of Schubert varieties in generalized flag varieties. In particular, the Möbius functions of these orderings are of interest since they (1) occur naturally in inversion formulas involving sums over Bruhat subintervals, and (2) provide topological information about the associated chain complexes (namely, reduced Euler characteristics for subintervals).

The Möbius function for the Bruhat order was first obtained by Verma [10], although his proof had a flaw that he later corrected in an unpublished paper (see the discussion in Section 8.5 of [7]). Deodhar subsequently proved a generalization covering the case of parabolic quotients [5]. Another way to obtain the Möbius function has been developed by Björner and Wachs [2] (see also [1] and [6]), and is based on a lexicographic shelling of the Bruhat order and its parabolic quotients. Kazhdan and Lusztig also point out (see Remark 3.3 of [9]) how to obtain the Möbius function for the full Bruhat orderings of finite Coxeter groups from basic properties of Kazhdan-Lusztig polynomials.

The goal of this paper is to derive these Möbius functions by a short, self-contained argument; it is noteworthy that the apparent lack of such an approach has been mentioned in the literature (see Section 6 of [4]). For the full Bruhat order, once the

This work was supported by NSF grant DMS-0532088.

J. R. Stembridge $(\triangle)$

Department of Mathematics, University of Michigan, Ann Arbor, Michigan 48109-1043 
preliminaries in Sections 1 and 2 are out of the way, the proof amounts to an easy calculation in the 0-Hecke algebra (see Lemma 3.2). In the symmetric group case, a similar calculation involving divided difference operators has been given by Lascoux (Lemma 1.13 of [8]).

For parabolic quotients, we use a similarly pleasant calculation in a module for the 0 -Hecke algebra (see Lemma 4.3). We have not seen this calculation elsewhere; the only previous derivations of the Möbius function in the parabolic case we have seen are the ones based on the shelling argument of Björner-Wachs, and Deodhar's original proof.

\section{The Bruhat order}

Let $(W, S)$ be a Coxeter system. For each $w \in W$, we let $\ell(w)$ denote the minimum length among all expressions $w=s_{1} \cdots s_{l}\left(s_{i} \in S\right)$. By Tits' Theorem [3, IV.5], one knows that any reduced (i.e., minimum-length) expression for $w$ may be transformed into any other by a sequence of braid relations; i.e., relations of the form

$$
\begin{aligned}
(s t)^{m} & =(t s)^{m} \quad \text { if } s t \text { has order } 2 m, \\
(s t)^{m} s & =(t s)^{m} t \quad \text { if } s t \text { has order } 2 m+1
\end{aligned}
$$

for all $s, t \in S$ such that $s t$ has finite order in $W$.

Let ' $\leq$ ' denote the Bruhat ordering of $W$. The most suitable definition of this ordering for our purposes is based on the Subword Property; i.e.,

$$
\begin{aligned}
& x \leq w \quad \Leftrightarrow \quad \begin{array}{l}
\text { for some (equivalently, every) reduced expression } w=s_{1} \cdots s_{l}, \\
\text { there is a reduced subword } x=s_{i_{1}} \cdots s_{i_{k}}\left(1 \leq i_{1}<\cdots<i_{k} \leq l\right) .
\end{array}
\end{aligned}
$$

The lack of dependence on the chosen reduced expression for $w$ (and thus, transitivity) is an easy consequence of Tits' Theorem. Indeed, if two reduced expressions for $w$ differ by a single braid relation, then the corresponding sets of reduced subwords are identical except for those that involve taking every term that participates in the braid relation.

The following result is a well-known recursive characterization of the Bruhat ordering (e.g., see [10] or Theorem 1.1 of [5]). We include a proof for the sake of completeness.

Proposition 1.1. For all $x, w \in W$ and $s \in S$ such that $\ell(s w)<\ell(w)$,

$$
x \leq w \text { if and only if }\left\{\begin{array}{l}
\ell(s x)<\ell(x) \text { and } s x \leq s w, \quad \text { or } \\
\ell(s x)>\ell(x) \text { and } x \leq s w .
\end{array}\right.
$$

Proof: Since $\ell(s w)<\ell(w)$, there is a reduced expression of the form $w=s_{1} \cdots s_{l}$ with $s_{1}=s$. In particular, $s w \leq w$. Thus if $x \leq s w$, then transitivity implies $x \leq w$. If $s x \leq s w$ and $\ell(s x)<\ell(x)$, then there is a reduced expression $s x=s_{i_{1}} \cdots s_{i_{k}}$ with 
$i_{1}>1$, and $x=s_{1} s_{i_{1}} \cdots s_{i_{k}}$ is a (necessarily) reduced expression that occurs as a subword of $s_{1} \cdots s_{l}$; i.e., $x \leq w$. Conversely, suppose $x \leq w$ and $x=s_{i_{1}} \cdots s_{i_{k}}$ is reduced. If $\ell(s x)>\ell(x)$, then $i_{1}>1$ and this expression occurs as a subword of $s_{2} \cdots s_{l}$ (i.e., $x \leq s w)$. If $\ell(s x)<\ell(x)$, then by the Exchange Property [3, IV.5], a reduced expression for $x$ may be obtained by deleting a single term from $s_{i_{1}} \cdots s_{i_{k}}$ and prepending $s=s_{1}$, so $s x$ has a reduced expression that occurs as a subword of $s_{2} \cdots s_{l}$; i.e., $s x \leq s w$.

For the remainder of this paper, the definition of the Bruhat order could be discarded, saving only the above result and the fact that 1 is the minimum element. However, one should avoid the temptation to use Proposition 1.1 as the basis of a definition, since it would not be clear a priori that different choices for $s$ lead to consistent results.

\section{The 0-Hecke algebra}

Let $H$ denote the Iwahori-Hecke algebra associated to $(W, S)$ with parameter $q=0$. More explicitly, define $H$ to be the $\mathbf{Q}$-algebra with unit element 1 , generators $\left\{v_{s}\right.$ : $s \in S\}$, quadratic relations

$$
v_{s}^{2}=-v_{s} \quad(s \in S)
$$

and the braid relations of $(W, S)$; i.e.,

$$
\begin{aligned}
\left(v_{s} v_{t}\right)^{m} & =\left(v_{t} v_{s}\right)^{m} & & \text { if } s t \text { has order } 2 m, \\
\left(v_{s} v_{t}\right)^{m} v_{s} & =\left(v_{t} v_{s}\right)^{m} v_{t} & & \text { if } s t \text { has order } 2 m+1
\end{aligned}
$$

for all $s, t \in S$ such that $s t$ has finite order in $W$.

Given the braid relations, Tits' Theorem implies that for each group element $w \in W$, there is a well-defined element $v_{w} \in H$ such that

$$
v_{w}=v_{s_{1}} \cdots v_{s_{l}}
$$

for all reduced expressions $w=s_{1} \cdots s_{l}\left(s_{i} \in S\right)$.

The following is the $q=0$ case of a standard but nontrivial fact about IwahoriHecke algebras that is often the first thing one proves when they are introduced (e.g., see Chapter 7 of [7]). The $q=0$ case is much easier, and since it is essentially the only feature of $H$ that we need, we include a proof.

Proposition 2.1. The elements $\left\{v_{w}: w \in W\right\}$ form a basis for $H$.

Proof: It is clear that the alleged basis spans $H$. To establish independence, let us introduce linear operators $A_{s}(s \in S)$ on the group algebra $\mathbf{Q} W$ by setting

$$
A_{s}(w)=\left\{\begin{array}{cl}
s w & \text { if } \ell(s w)>\ell(w) \\
w & \text { if } \ell(s w)<\ell(w) .
\end{array}\right.
$$


It is immediate that $A_{s}^{2}=A_{s}$, and we claim that these operators also satisfy the braid relations of $(W, S)$. Indeed, if $s t$ has order $2 m$ or $2 m+1$ in $W(s, t \in S)$, then $(s t)^{m}$ or $(s t)^{m} s$ is the longest element of the dihedral subgroup $\langle s, t\rangle$, and it follows from the well-known structure of parabolic cosets (e.g., see Exercise IV.1.3 of [3]) that $\left(A_{s} A_{t}\right)^{m}(w)$ or $\left(A_{s} A_{t}\right)^{m} A_{s}(w)$ is the longest element of the coset $\langle s, t\rangle w$. The latter is clearly symmetric in $s$ and $t$, and hence the corresponding braid relation holds. It follows that $v_{s} \mapsto-A_{s}$ defines a representation of $H$ as an algebra of endomorphisms of $\mathbf{Q} W$. Equivalently, $\mathbf{Q} W$ is an $H$-module. Since $v_{w}$ maps the unit element of $\mathbf{Q} W$ to $\pm w$ under this action, the independence follows.

\section{The Möbius function}

Let $\mu$ denote the Möbius function for the Bruhat order; i.e., the unique integer function on pairs $x \leq w$ in $W$ such that $\mu(w, w)=1$ and $\sum_{x \leq y \leq w} \mu(y, w)=0$ if $x<w$.

Theorem 3.1. (Verma). We have $\mu(x, w)=(-1)^{\ell(w)-\ell(x)}$ for all $x \leq w$ in $W$.

Our proof follows from an easy calculation in $H$.

Lemma 3.2. If $w=s_{1} \cdots s_{l}$ is reduced, then

$$
\left(v_{s_{1}}+1\right) \cdots\left(v_{s_{l}}+1\right)=\sum_{x \leq w} v_{x}
$$

Proof: The case $w=1$ is trivial, so assume $l>0$ and set $s=s_{1}$. By induction we may assume the result to be true for $s w=s_{2} \cdots s_{l}$, and hence

$$
\left(v_{s_{1}}+1\right) \cdots\left(v_{s_{l}}+1\right)=\sum_{x \leq s w}\left(v_{s}+1\right) v_{x}
$$

If there is a reduced expression for $x$ starting with $s$, then $\left(v_{s}+1\right) v_{x}=\left(v_{s}+1\right) v_{s} v_{s x}=$ 0 . If there is no such expression, then $\ell(s x)>\ell(x)$ and $v_{s} v_{x}=v_{s x}$, whence

$$
\sum_{x \leq s w}\left(v_{s}+1\right) v_{x}=\sum_{x \leq s w, \ell(s x)>\ell(x)} v_{s x}+v_{x} .
$$

The above sum has exactly one copy of $v_{y}$ for each $y \leq w$, by Proposition 1.1.

Let us introduce a second set of generators for $H$ by defining

$$
u_{s}:=v_{s}+1 \quad(s \in S)
$$

In these terms, Lemma 3.2 may be restated as the identity

$$
u_{w}=\sum_{x \leq w} v_{x}
$$


where $u_{w}:=u_{s_{1}} \cdots u_{s_{l}}$ for any reduced expression $w=s_{1} \cdots s_{l}$. Note that the right side of (1) depends only on $w$, so $u_{w}$ does not depend on the choice of reduced expression.

Lemma 3.3. The map $v_{s} \mapsto-u_{s}(s \in S)$ defines a ring involution on $H$.

Proof: If $w$ is the longest element of some (finite) parabolic subgroup generated by a pair $s, t \in S$, then the expression-independence of $u_{w}$ implies that $u_{s}$ and $u_{t}$ satisfy the corresponding braid relation of $(W, S)$. Also, it is easy to check that $u_{s}^{2}=u_{s}$, so the elements $\left\{-u_{s}: s \in S\right\}$ obey the defining relations of $H$, and thus there is a unique automorphism of $H$ such that $v_{s} \mapsto-u_{s}$ for all $s \in S$. Since $u_{s}=\left(v_{s}+1\right) \mapsto$ $1-u_{s}=-v_{s}$, this automorphism is an involution.

Proof of Theorem 3.1. Applying the involution of Lemma 3.3 to (1), we obtain

$$
v_{w}=\sum_{x \leq w}(-1)^{\ell(w)-\ell(x)} u_{x} .
$$

The fact that this inverts (1) shows that $(x, w) \mapsto(-1)^{\ell(w)-\ell(x)}$ satisfies the defining property of the Möbius function.

\section{The parabolic case}

Let $W_{J}$ denote the parabolic subgroup of $W$ generated by some fixed $J \subseteq S$, and let

$$
W^{J}=\{w \in W: \ell(w s)>\ell(w) \text { for all } s \in J\}
$$

denote the unique set of minimal coset representatives for $W / W_{J}$ (Exercise IV.1.3 in [3]). It is well-known (ibid) that for all $x \in W^{J}$ and $y \in W_{J}$, one has

$$
\ell(x y)=\ell(x)+\ell(y) .
$$

Lemma 4.1. For all $x \in W^{J}$ and $s \in S$, either $s x \in W^{J}$, or $\ell(s x)>\ell(x)$ and $s x=x t$ for some $t \in J$.

Proof: If $s x \notin W^{J}$, then $\ell(s x t)<\ell(s x)$ for some $t \in J$. If $\ell(s x)<\ell(x)$, this forces $\ell(x t)<\ell(x)$ and contradicts having $x \in W^{J}$. Hence $\ell(s x)>\ell(x)$ and $s x$ has a reduced expression of the form $s s_{1} \cdots s_{l}$. By the Exchange Property, it is possible to transform this into another reduced expression for $s x$ by appending $t$ and deleting either $s$ or some $s_{i}$. In the former case, $s x=x t$; in the latter, we obtain $\ell(x t)<\ell(x)$, a contradiction.

Define a binary relation on $W^{J}$ by declaring

$$
x \preccurlyeq w \quad \Leftrightarrow \quad x \leq w \text { and } x t \nless w \quad \text { for all } t \in J .
$$

Unlike the Bruhat order (the case $J=\varnothing$ ), this relation need not be transitive. 
Lemma 4.2. For all $x, w \in W^{J}$ and $s \in S$ such that $\ell(s w)<\ell(w)$,

$$
x \preccurlyeq w \text { if and only if }\left\{\begin{array}{l}
\ell(s x)<\ell(x) \text { and } s x \preccurlyeq s w, \quad \text { or } \\
\ell(s x)>\ell(x), x \preccurlyeq s w \text { and } s x \in W^{J} .
\end{array}\right.
$$

Note that in the above context, Lemma 4.1 implies $s w \in W^{J}$.

Proof: Suppose $\ell(s x)<\ell(x)$. In that case, Proposition 1.1 implies $x \leq w$ if and only if $s x \leq s w$. We also have $s x \in W^{J}$ (Lemma 4.1), so for all $t \in J$ we have $\ell(s x t)<\ell(x t)$, and hence $x t \leq w$ if and only if $s x t \leq s w$ (Proposition 1.1); i.e., $x \preccurlyeq w$ if and only if $s x \preccurlyeq s w$.

The remaining possibility is that $\ell(s x)>\ell(x)$. In that case, we have $x \leq w$ if and only if $x \leq s w$, again by Proposition 1.1. If $s x \notin W^{J}$, then Lemma 4.1 implies $s x=x t$ for some $t \in J$. On the other hand, $x \preccurlyeq w$ implies $x \leq w$, and hence $x \leq s w$ and $s x=x t \leq w$ (for the latter, apply Proposition 1.1 to the pair $(s x, w)$ ), a contradiction. Thus we may add the condition $s x \in W^{J}$ to our assumptions about $x$. For all $t \in J$, it follows that $\ell(s x t)>\ell(x t)$, and thus $x t \leq w$ if and only if $x t \leq s w$; i.e., $x \preccurlyeq w$ if and only if $x \preccurlyeq s w$.

Recall from the proof of Proposition 2.1 that $\mathbf{Q} W$ may be viewed as an $H$-module in which $-v_{s}(w)=s w($ if $\ell(s w)>\ell(w))$ or $w($ if $\ell(s w)<\ell(w))$. We claim that

$$
M_{J}:=\operatorname{Span}\left\{w-w y: w \in W^{J}, y \in W_{J}\right\}
$$

is an $H$-submodule of $\mathbf{Q} W$. Indeed, given $w \in W^{J}$ and $y \in W_{J}$, consider $-v_{s}(w-$ $w y)$. If $s w \in W^{J}$ then (3) implies $\ell(s w)<\ell(w)$ if and only if $\ell(s w y)<\ell(w y)$, and hence $-v_{s}(w-w y)=w-w y$ or $s w-s w y$. Either way, $-v_{s}(w-w y) \in M_{J}$. By Lemma 4.1, the only other possibility is that $s w=w t$ for some $t \in J$, in which case $-v_{s}(w)=w t$ and $-v_{s}(w y)=w y^{\prime}$, where $y^{\prime} \in W_{J}$ is the longer of $t y$ or $y$. Hence

$$
-v_{s}(w-w y)=w t-w y^{\prime}=\left(w-w y^{\prime}\right)-(w-w t) \in M_{J}
$$

proving the claim. The quotient module $\mathbf{Q} W / M_{J}$ has a basis $\left\{[w]_{J}: w \in W^{J}\right\}$, where

$$
[w]_{J}:=w+M_{J}
$$

Furthermore, if $s w=w t$ for some $t \in J$ (i.e., $s w \notin W^{J}$ ), then $-v_{s}(w)=w t=w$ $\bmod M_{J}$, so $-v_{s}$ acts on this basis via the rule

$$
-v_{s}[w]_{J}= \begin{cases}{[s w]_{J}} & \text { if } s w \in W^{J} \text { and } \ell(s w)>\ell(w) \\ {[w]_{J}} & \text { otherwise. }\end{cases}
$$

Note that if $W_{J}$ is finite with longest element $z$, this is isomorphic to the action of $-v_{s}$ on the left ideal of $H$ generated by $v_{z}$, relative to the basis $\left\{(-1)^{\ell(w)} v_{w z}: w \in W^{J}\right\}$. 
The following calculation generalizes Lemma 3.2.

Lemma 4.3. For all $w \in W^{J}$, we have

$$
u_{w}[1]_{J}=\sum_{x \preccurlyeq w} v_{x}[1]_{J}=\sum_{x \preccurlyeq w}(-1)^{\ell(x)}[x]_{J} .
$$

Proof: The case $w=1$ is trivial, so assume there is some $s \in S$ such that $\ell(s w)<$ $\ell(w)$. In that case, we have $u_{w}=\left(v_{s}+1\right) u_{s w}$ and $s w \in W^{J}$, so by induction,

$$
u_{w}[1]_{J}=\left(v_{s}+1\right) u_{s w}[1]_{J}=\sum_{x \preccurlyeq s w}\left(v_{s}+1\right) v_{x}[1]_{J}=\sum_{x \preccurlyeq s w}(-1)^{\ell(x)}\left(v_{s}+1\right)[x]_{J} .
$$

If $\ell(s x)<\ell(x)$ or $s x \notin W^{J}$, then (4) implies $\left(v_{s}+1\right)[x]_{J}=0$, and hence

$$
u_{w}[1]_{J}=\sum_{x \preccurlyeq s w, \ell(s x)>\ell(x), s x \in W^{J}}(-1)^{\ell(s x)}[s x]_{J}+(-1)^{\ell(x)}[x]_{J} .
$$

Lemma 4.2 implies that this sum ranges over those $x$ such that $x \preccurlyeq w$ and $\ell(s x)>\ell(x)$, and at the same time, $y=s x$ ranges over those $y \preccurlyeq w$ such that $\ell(s y)<\ell(y)$.

Theorem 4.4 (Deodhar). As a subposet of $(W, \leq)$, the Möbius function for $W^{J}$ is

$$
\mu_{J}(x, w)= \begin{cases}(-1)^{\ell(w)-\ell(x)} & \text { if } x \preccurlyeq w, \\ 0 & \text { otherwise }\end{cases}
$$

Proof: Given $w \in W^{J}$, use (2) to apply $v_{w}$ to $[1]_{J}$ in $\mathbf{Q} W / M_{J}$, obtaining

$$
(-1)^{\ell(w)}[w]_{J}=v_{w}[1]_{J}=\sum_{x \leq w}(-1)^{\ell(w)-\ell(x)} u_{x}[1]_{J}
$$

If $x \notin W^{J}$, then $u_{x}=u_{x s} u_{s}$ for some $s \in J$, and (4) implies $u_{s}[1]_{J}=0$, so

$$
[w]_{J}=\sum_{x \leq w, x \in W^{J}}(-1)^{\ell(x)} u_{x}[1]_{J} .
$$

Inverting this relationship, one sees that $\mu_{J}(x, w)$ is the coefficient of $[x]_{J}$ in the expansion of $(-1)^{\ell(w)} u_{w}[1]_{J}$, and that Lemma 4.3 provides this expansion.

\section{References}

1. A. Björner and F. Brenti, Combinatorics of Coxeter Groups, Springer, New York, 2005.

2. A. Björner and M. Wachs, "Bruhat order of Coxeter groups and shellability," Adv. in Math. 43 (1982) 87-100. 
3. N. Bourbaki, Groupes et Algèbres de Lie, Chp. IV-VI, Masson, Paris, 1981.

4. F. Brenti, S. Fomin, and A. Postnikov, "Mixed Bruhat operators and Yang-Baxter equations for Weyl groups," Internat. Math. Res. Notices 1999, 419-441.

5. V.V. Deodhar, "Some characterizations of Bruhat ordering on a Coxeter group and determination of the relative Möbius function," Invent. Math. 39 (1977) 187-198.

6. M.J. Dyer, "Hecke algebras and shellings of Bruhat intervals," Compositio Math. 89 (1993) 91-115.

7. J.E. Humphreys, Reflection Groups and Coxeter Groups, Cambridge Univ. Press, Cambridge, 1990.

8. A. Lascoux, "Anneau de Grothendieck de la variété de drapeaux," in "The Grothendieck Festschrift, vol. III", pp. 1-34, Progr. Math. 88, Birkhäuser, Boston, 1990.

9. D. Kazhdan and G. Lusztig, "Representations of Coxeter groups and Hecke algebras," Invent. Math. 53 (1979) 165-184.

10. D.-N. Verma, "Möbius inversion for the Bruhat ordering on a Weyl group," Ann. Sci. École Norm. Sup. 4 (1971) 393-398. 